\title{
Study on Optimization of Logistics Distribution Routes Based on Opposi- tion-based Learning Particle Swarm Optimization Algorithm
}

\author{
Liu Xiao-jun and Zhang Bin*
}

\author{
Department of Logistics and Information Management, Zhuhai College of Jilin University, Zhuhai, China
}

\begin{abstract}
In view of shortcomings of the particle swarm optimization algorithm such as poor late optimization ability and proneness to local optimization etc, this paper proposes an opposition-based learning particle swarm optimization (OBLPSO) algorithm for the optimization of logistics distribution routes, firstly, establishes a logistics distribution route optimization mathematical model, and then solves through collaboration and information exchange among particles, introduces an opposition-based learning mechanism to improve particle swarm optimization ability and convergence rate, and finally conducts simulation test on the performance of OBLPSO algorithm on Matlab 2012 platform. The simulation results show that OBLPSO algorithm can be used to obtain logistics distribution solutions with short time and rational routes and thus has certain practical value, compared with other logistics distribution route optimization algorithms.
\end{abstract}

Keywords: Opposition-based learning, particle swarm optimization, logistics distribution, route selection.

\section{INTRODUCTION}

Logistics distribution is to deliver goods to specified destination on time according to customer requirements; distribution route optimization is the key of logistics distribution, and rational selection of logistics distribution routes can save costs and improve economic benefits; thus, logistics distribution route optimization becomes a hot but difficult topic in study on logistics field [1].

Logistics distribution route optimization is a multiconstraint and combinatorial optimization problem, and belongs to Non-deterministic Polynomial Complete (NPC) difficulty; with the rapid development of logistics scale, manual arrangement of logistics routes can hardly meet modern logistics requirements, and distribution routes are currently arranged by computer automatically [2]. With respect to distribution route optimization, scholars spend a lot of time and energy on in-depth and extensive researches, and put forward three types of distribution route optimization methods namely precise methods, heuristic algorithms and swarm intelligence algorithms etc. Precise methods include dynamic programming method and branch \& bound method etc, could reach optimal solutions to small-scale logistics distribution route optimization problems, but have great calculation complexity and low solving efficiency for modern large-scale logistics distribution route optimization problems [3]. Heuristic algorithms include construction algorithm and two-stage method, and have improved solving efficiency compared with precise methods, but can hardly get optimal solutions to logistics distribution routes [4]. Swarm intelligence algorithms mainly include genetic algorithm, particle

*Address correspondence to this author at the Department of Logistics and Information Management, Zhuhai College of Jilin University, Zhuhai, China; Tel: +860000000000; E-mail: Zhangbin1218@sina.com swarm optimization algorithm, frog leaping algorithm and neural network algorithm etc, which have advantages such as rapid search speed etc and become main methods for solving logistics distribution problems [5-8]. Among these methods, particle swarm optimization (PSO) algorithm has advantages including strong global optimization ability and simple \& easy implementation etc, and thus is the most widely used for logistics distribution route optimization; however, PSO algorithm has shortcomings such as slow late speed and proneness to local optimization etc, and thus scholars put forward many logistics distribution route optimization methods to improve PSO algorithm and gain a better optimal solution to logistics distribution route [9]. However, further improvement is still needed and new ideas shall be introduced for better logistics distribution route solutions. In 2005, Tizhoosh put forward opposition-based learning (OBL) theory, and believed that the optimal solution would finally be found through constant iteration by swarm intelligence algorithm if taking random predictive value as the initial swarm; thus, random predictive value was vital for swarm intelligence algorithm, rapid convergence rate would be reached if this value was close to the optimal solution, while there would be long time and slow speed if not; however, the efficiency of swarm intelligence algorithm would be substantially improved if both current solution and inverse solution were both searched in swarm intelligence algorithm search and the optimal solution was selected to be predictive value according to results [10]. Based on this, with respect to shortcomings of PSO algorithm, "OBL theory" is introduced to generate an OBLPSO algorithm, and this algorithm is used to solve logistics distribution route problem, and the performance of this algorithm is tested through simulation experiment. 


\section{LOGISTICS DISTRIBUTION ROUTE OPTI- MIZATION MATHEMATICAL MODEL}

\subsection{Distribution Route Problem}

Set that a logistics distribution network contains a total of $\mathrm{M}$ customer sites, and thus the locations and goods demands of these customer sites are known; the logistics distribution center has a total of $\mathrm{K}$ distribution vehicles, each vehicle $\mathrm{k}$ has given and known maximum carrying capacity $\mathrm{Pk}$ $(\mathrm{k}=1,2, \ldots, \mathrm{K})$ and starts from and returns to the distribution center. The objective of logistics distribution route optimization is to find an optimal solution to total costs (such as distance and time etc) while meeting the following constraints:

(1) The sum of goods demand on each route shall not exceed the maximum vehicle carrying capacity.

(2) The location of logistics distribution center is fixed and known.

(3) Service for one customer site can only be provided by one vehicle.

(4) Distribution route length shall not exceed the maximum distance every time a vehicle drives [10].

\subsection{Logistics Distribution Route Optimization Mathe- matical Model}

$b_{0,0}$ denotes the distribution center of logistics network, $b_{i, j}$ denotes the distance between customer $\mathrm{i}$ and customer $\mathrm{j}$, and $i, j=0,1, \mathrm{~K}, M, n_{k}$ denotes the number of customers that vehicle $\mathrm{k}$ distributes. Thus, the mathematical model of logistics distribution route optimization is:

$$
\operatorname{MinF}=\sum_{k=1}^{K}\left(\sum_{i=1}^{n_{k}} b_{r_{k}^{i-1}, r_{k}^{i}}+b_{r_{k}^{n k}, 0}\right) \times \operatorname{sgn}\left(n_{k}\right)
$$

Where:

$$
\operatorname{sgn}\left(n_{k}\right)= \begin{cases}1 & n_{k}=0 \\ 0 & n_{k} \geq 1\end{cases}
$$

Constraints of logistics distribution route optimization are:

$$
\left\{\begin{array}{l}
\sum_{i=1}^{n_{k}} p_{r_{k}^{i}} \leq p k ; n_{k} \neq 0 \\
\sum_{i=1}^{n_{k}} b_{r_{k}^{i-1}, r_{k}^{i}}+b_{r_{k}^{n_{k}}, 0} \leq B_{k} ; n_{k} \neq 0 \\
R_{k 1} \mathrm{I} R_{k 2}=\phi, k_{1} \neq k_{2} \\
\bigcup_{k=1}^{K} R_{k}=\{1,2, \mathrm{~L}, M\} ; 0 \leq n_{k} \leq M
\end{array}\right.
$$

Where, $R_{k}$ denotes customer set of vehicle $\mathrm{k} ; B_{k}$ denotes the maximum travel distance of vehicle $\mathrm{k}$; and $r_{k}^{j}$ denotes the order of customer in distribution route.

According to formula (1), the logistics distribution process has two objectives. The problem which requires mini- mum distribution vehicles, the shortest distribution route and delivery of goods to destination within the specified time is a multi-constraint and multi-objective combinatorial optimization problem. As swarm intelligence optimization algorithm has certain advantage in solving combinatorial optimization problem, this paper adopts OBLPSO algorithm to solve formula (1) and find a logistics distribution solution that meets all constraints.

\section{OBLPSO ALGORITHM FOR LOGISTICS DIS- TRIBUTION ROUTE OPTIMIZATION}

\subsection{Concepts Related to OBL}

The basic idea of OBL algorithm is to obtain current optimal value by considering and comparing current estimated value and inverse estimated value of variable.

Definition 1 Any real number in $x \in[a, b]$, define $\hat{x}=a+b-x$ as the inverse point of $\mathrm{x}$.

Definition $2 X=\left[x_{1}, x_{2}, \mathrm{~L}, x_{D}\right]$ is a point in Ddimension space, where $x_{i} \in\left[a_{i}, b_{i}\right]$, and define $\hat{X}=\left[\hat{x}_{1}, \hat{x}_{2}, \mathrm{~L}, \hat{x}_{D}\right]$ as the inverse point of $\mathrm{X}$ with element of $\hat{x}_{i}=a_{i}+b_{i}-x_{i}$.

Definition 3 Assume that $X=\left[x_{1}, x_{2}, \mathrm{~L}, x_{D}\right]$ is a point in D-dimension space, $\mathrm{f}()$ is a fitness function to measure this point, $\hat{X}=\left[\hat{x}_{1}, \hat{x}_{2}, \mathrm{~L}, \hat{x}_{D}\right]$ is the inverse point of $\mathrm{x}$, such as $f(\hat{X})<f(X)$, then, replace $\mathrm{X}$ with $\hat{X}$, otherwise, continue using point $X$, and obtain a relatively optimal solution through fitness values of evaluation point $X$ and inverse point $\hat{X}$.

\subsection{OBLPSO Algorithm}

Set the flying speed and location of particle $i$ as: $v_{i}=\left(v_{i 1}, v_{i 2}, \mathrm{~L}, v_{i D}\right)^{T}$ and $x_{i}=\left(x_{i 1}, x_{i 2}, \mathrm{~L}, x_{i D}\right)^{T}$ respectively, the previous optimal location of particle $i$ is $p_{i}=\left(p_{i 1}, p_{i 2}, \mathrm{~L}, p_{i D}\right)$, the optimal swarm location is $p_{g}=\left(p_{g 1}, p_{g 2}, \mathrm{~L}, p_{g D}\right)$, and the location and speed update mode of particle $\mathrm{i}$ are:

$$
\begin{gathered}
v_{i d}(t+1)=w v_{i d}+c_{1} \operatorname{rand}()\left(p_{i d}-X_{i d}(t)\right)+ \\
c_{2} \operatorname{rand}()\left(p_{g d}-X_{i d}(t)\right) \\
x_{i d}(t+1)=x_{i d}(t)+v_{i d}(t+1)
\end{gathered}
$$

Where, $c_{1}$ and $c_{2}$ are acceleration coefficients; rand() is a random number in $[0,1]$; $\mathrm{t}$ is current iteration times; and $\omega$ is inertia weight which is defined as follows:

$$
\omega=\omega_{\max }-\frac{i t e r}{i t e r}\left(\omega_{\max }-\omega_{\min }\right)
$$

Where, $\omega_{\max }$ is the maximum weight coefficient; and $\omega_{\min }$ is the minimum weight coefficient. 
Table 1. Specific particle coding.

\begin{tabular}{|c|c|c|c|c|c|c|c|}
\hline Customer Site & $\mathbf{1}$ & $\mathbf{2}$ & $\mathbf{3}$ & $\mathbf{4}$ & $\mathbf{5}$ & $\mathbf{6}$ & $\mathbf{7}$ \\
\hline \hline Zix & 2.8 & 1.3 & 3.3 & 2.6 & 1.7 & 3.6 & 2.4 \\
\hline Ziy & 2.6 & 0.8 & 1.9 & 3.5 & 4.7 & 2.9 & 2.0 \\
\hline
\end{tabular}

According to particle decoding mode, situation of each vehicle in each customer site is shown in Table 2.

Table 2. Specific particle decoding.

\begin{tabular}{|c|c|c|c|c|c|c|c|}
\hline Customer Site & $\mathbf{1}$ & $\mathbf{2}$ & $\mathbf{3}$ & $\mathbf{4}$ & $\mathbf{5}$ & $\mathbf{6}$ & $\mathbf{7}$ \\
\hline \hline $\operatorname{int}(\mathrm{Zix})$ & 2 & 1 & 3 & 2 & 1 & 3 \\
\hline Ziy & 2.6 & 0.8 & 1.9 & 3.5 & 4.7 & 2.9 & 2.0 \\
\hline
\end{tabular}

A particle of swarm has growing information demand with its continuous evolution and growth, and mere reliance on $\mathrm{p}_{\mathrm{i}}$ and $\mathrm{p}_{\mathrm{g}}$ "extreme values" could not meet such demand. Thus, the ability of other particles in the swarm to explore new areas shall be strengthened. Based on the above analysis, OBLPSO algorithm is put forward.

On the premise of tracking $p_{i}$ and $p_{g}$ in each iteration of random particle, another new extreme values shall be tracked as well, namely the worst solution $\mathrm{pp}_{\mathrm{i}}$ of particle itself and the worst solution $\mathrm{pp}_{\mathrm{g}}$ occurred currently in the entire swarm. Thus, this paper introduces the inverse particle $\stackrel{\mathrm{a}}{P} P_{g}$ of the worst global $\mathrm{pp}_{\mathrm{g}}$ in iteration mechanism as a new learning factor, and the specific iteration mechanism is as follows:

$$
\begin{aligned}
& v_{i d}(t+1)=w v_{i d}+c_{1} \operatorname{rand}()\left(p_{i d}-X_{i d}(t)\right)+ \\
& c_{2} \operatorname{rand}()\left(p_{g d}-X_{i d}(t)\right)+c_{3} \operatorname{rand}()\left(\stackrel{\mathrm{a}}{P} P_{g}-X_{i d}(t)\right)
\end{aligned}
$$

The experiment proves that $\stackrel{\mathrm{a}}{\mathrm{P}} P_{\mathrm{g}}$ does not always guide particle swarm to the optimal value, i.e. some situations would affect global learning ability of particle. Thus, improvement is made based on inverse point $\stackrel{\mathrm{a}}{P} P_{g}$.

$$
\stackrel{\mathrm{a}}{P} P_{g}=\left\{\begin{array}{l}
a+b-\left(m_{1} p p_{g}+m_{2} p_{i}\right), \text { ifrand }()<p \\
0, \text { otherwise }
\end{array}\right.
$$

Where, $\mathrm{m}_{1}$ and $\mathrm{m}_{2}$ are random numbers in $[0,1], \mathrm{m}_{1}+\mathrm{m}_{2}=1$, and $\mathrm{p}$ is the possibility of changing into OBL algorithm and is defined as follows:

$$
p=p_{\max }-t \times\left(p_{\max }-p_{\min }\right) / T
$$

Where, $p_{\max }$ and $p_{\min }$ are scopes of possibility $\mathrm{p}$; and $\mathrm{t}$ is current iteration times.

OBLPSO algorithm not only adds particle diversity to avoid local optimization problem, but also narrows the search space to improve particle search speed and avoid over learning phenomenon.

\subsection{Particle Coding Mode}

During logistics distribution route optimization, the correspondence of particle location to optimization solution is the most crucial. This paper builds a $2 n$-dimension space for distribution to $\mathrm{n}$ customer sites, each of which is corresponding to service vehicles and execution order, and thus particle $i$ divides $2 n$-dimension vector $Z$ into two $n$-dimension vectors $Z_{i x}$ and $Z_{i y}$, which denote vehicle number and route order respectively.

\subsection{Particle Decoding Mode}

(1) Firstly, take the integer of $Z_{i x}$ of particle $i$ to get corresponding vehicle number $\mathrm{j}$ distributed to customer $\mathrm{i}$.

(2) Then, determine route order of vehicle $\mathrm{j}$ according to size order of element $\mathrm{Z}_{\mathrm{iy}}$.

Assume that a distribution center has 7 customers and 3 vehicles, then code $\mathrm{Z}$ of particle $\mathrm{i}$ is shown in Table $\mathbf{1}$.

Then, travel routes of vehicles corresponding to particle are $(0$ denotes the distribution center $)$ : vehicle 1: $0 \rightarrow 2 \rightarrow 4 \rightarrow 0$; vehicle $2: 0 \rightarrow 1 \rightarrow 5 \rightarrow 7 \rightarrow 0 ;$ and vehicle 3 : $0 \rightarrow 3 \rightarrow 6 \rightarrow 0$.

\subsection{Solving Steps of Logistics Distribution Route Opti- mization}

(1) Set parameters for OBLPSO algorithm particle swarm algorithm.

(2) Initialize particle swarm, and each particle denotes a potential feasible logistics distribution route.

(3) Decode each particle, calculate the shortest route of each distribution solution and take it as the particle fitness function value.

(4) Compare each particle location with previous optimal values of individuals and the swarm, and update previous optimal values for individuals and the swarm according to results.

(5) Generate a value in $[0,1]$ randomly, calculate the possibility $\mathrm{p}$ through formula (9), and go to step (6) if this ran- 
dom value is greater than $\mathrm{p}$, otherwise, enter OBL specifically as follows:

1) Calculate the inverse point of this particle by OBL mechanism based on crossover factor;

2) Calculate the adaptive values of particle $i$ and inverse particle which is based on crossover factor, compare whether the adaptive value of inverse particle is superior to that of particle $\mathrm{i}$, select the optimal particle to replace $\mathrm{X}$, otherwise, continue using point $X$.

(6) Update the location and speed of each particle according to formula (5) and (6).

(7) If the end condition is met, take the optimal particle location as the optimal solution, otherwise, go to step (3) and continue to optimize.

(8) Get the optimal logistics distribution route solution based on the optimal solution.

\section{SIMULATION EXPERIMENT}

\subsection{Classic Function Test}

Two types of classic standard multimodal functions are selected for the test experiment, and compare the test results with those obtained by PSO algorithm. These two types of classic test functions are specifically as follows:

(1) Sphere function

$$
f(x)=\sum_{i=1}^{n} x_{i}^{2}
$$

(2) Griewank function

$$
f(x)=1 / 4000 \sum_{i=1}^{n}\left(x_{i}\right)^{2}-\prod_{i=1}^{n} \cos \left(\frac{x_{i}}{\sqrt{i}}\right)+1
$$

Fig. (1) shows fitness log value evolution curves of 2 test functions (notes: this paper takes the base-10 logarithm of fitness values of functions for the convenience of display and observation of evolution curves). In the figure, the solid line is the convergence curve of PSO algorithm, while the dotted line is the convergence curve of OBLPSO algorithm. As can be known from Fig. (1), OBLPSO algorithm has a significantly superior convergence rate to PSO algorithm, and thus avoids the shortcoming of PSO namely local optimization problem. This is because OBLPSO contains OBL, which makes OBLPSO better than traditional PSO algorithm in terms of global search ability, convergence precision and speed.

\subsection{Simulation Test of Logistics Distribution Route Op- timization}

A company has one logistics distribution center and five goods transport vehicles (carrying capacity of each vehicle is $1 \mathrm{t}$ ), and needs to deliver goods to eight customer sites. Coordinates and goods demand of each customer site are shown in table 3 ( 0 denotes the distribution center and 1-8 are customer sites).

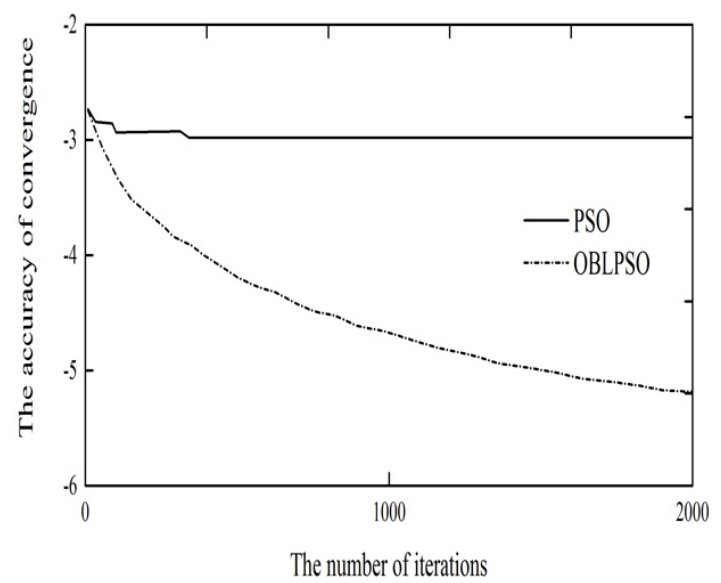

(a) Convergence Curve of Sphere Function

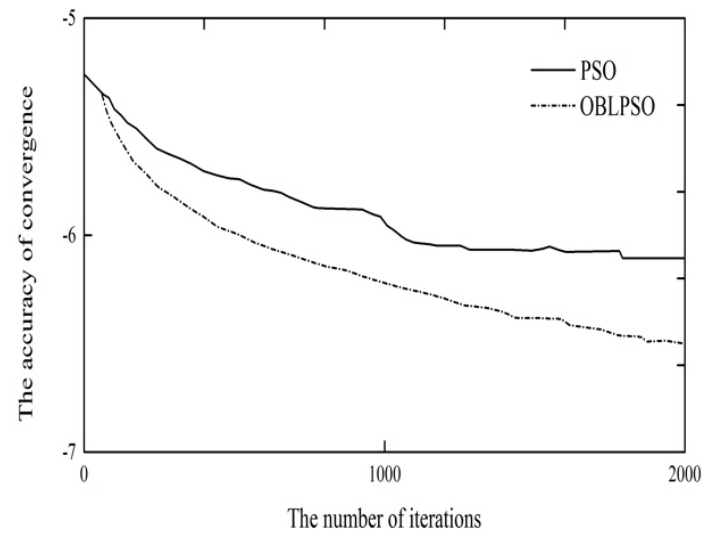

(b) Convergence Curve of Griewank Function Fitness

Fig. (1). Comparison of convergence performance between OBLPSO and PSO algorithm.

Table 3. Coordinates and goods demands of customers.

\begin{tabular}{|c|c|c|}
\hline Customer No. & Coordinates & Goods Demand \\
\hline \hline 0 & $(40,40)$ & \\
\hline 1 & $(10,20)$ & 1.30 \\
\hline 2 & $(15,50)$ & 2.38 \\
\hline 3 & $(30,40)$ & 1.77 \\
\hline 4 & $(40,60)$ & 2.40 \\
\hline 5 & $(38,10)$ & 1.81 \\
\hline 6 & $(50,45)$ & 1.22 \\
\hline 7 & $(70,20)$ & 1.81 \\
\hline 8 & $(60,70)$ & 1.20 \\
\hline
\end{tabular}

Parameters of OBLPSO algorithm are: swarm scale $\mathrm{N}=20$, the maximum evolution algebra $\mathrm{T}=500, \mathrm{c} 1=\mathrm{c} 2=1.5$, c3=1.0. Solve logistics distribution route optimization problem in Table 3 by PSO algorithm and OBLPSO algorithm respectively, and results are shown in (Figs. $\mathbf{2}$ and $\mathbf{3}$ ). 


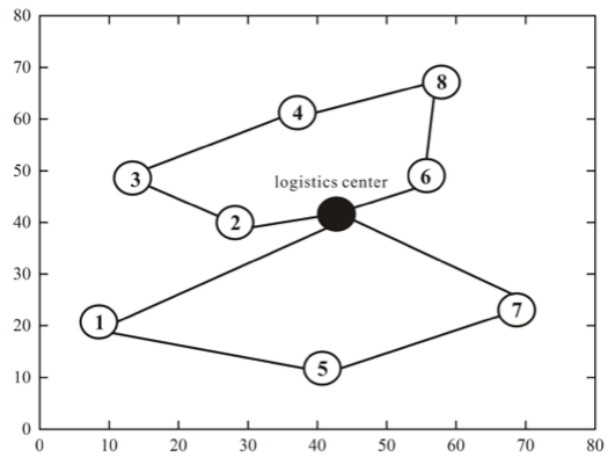

Fig. (2). The optimal logistics distribution route of PSO algorithm.

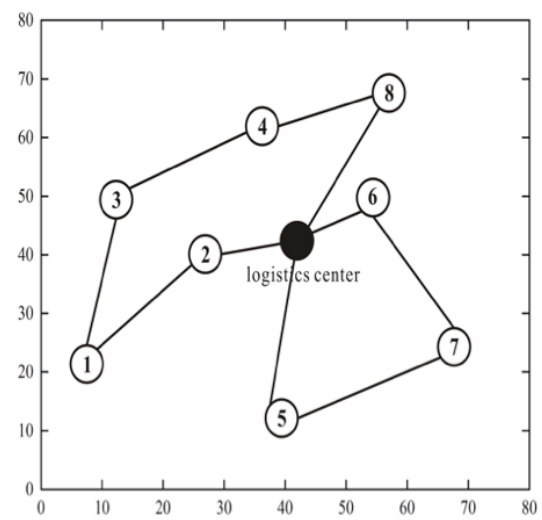

Fig. (3). The optimal logistics distribution route of OBLPSO algorithm.

From Fig. (2), there are 2 logistics distribution routes by PSO algorithm: route 1 is $0 \rightarrow 1 \rightarrow 5 \rightarrow 7 \rightarrow 0$ with total distribution route length of $135.36 \mathrm{~km}$ and route 2 is $0 \rightarrow 2 \rightarrow 3 \rightarrow 4 \rightarrow 8 \rightarrow 6 \rightarrow 0$ with total distribution route length of $156.98 \mathrm{~km}$, and thus the total route length is $292.34 \mathrm{~km}$.

From Fig. (3), there are 2 logistics distribution routes by OBLPSO algorithm: route 1 is $0 \rightarrow 2 \rightarrow 1 \rightarrow 3 \rightarrow 4 \rightarrow 8 \rightarrow 0$ with total distribution route length of $125.73 \mathrm{~km}$ and route 2 is $0 \rightarrow 5 \rightarrow 7 \rightarrow 6 \rightarrow 0$ with total distribution route length of $123.09 \mathrm{~km}$, and thus the total route length is $248.82 \mathrm{~km}$.

By comparing results of Fig. (2) and Fig. (3), logistics distribution route solution obtained by OBLPSO algorithm is superior to that obtained by PSO algorithm, mainly because of tracking of both global optimization and the inverse particle of the worst global particle during iteration of OBPSO algorithm and rapid convergence rate, which avoids effectively local optimization, prevents premature convergence and improves the search efficiency.

\section{CONFLICT OF INTEREST}

The authors confirm that this article content has no conflict of interest.

\section{ACKNOWLEDGEMENTS}

Declared none.

\section{REFERENCES}

[1] Y. Y. Shiu, and K. C. Chi, "A genetic algorithm that adaptively mutates and never revisits," IEEE Transactions on Evolutionary Computation, vol. 13, no. 2, pp. 454-458, 2009.

[2] L.Y. Tseng, and Y.T. Lin, "A hybrid genetic local search algorithm for the permutation flow shop scheduling problem," European Journal of Operational Research, vol. 198, no.1, pp. 84-92, 2009.

[3] X. Zhang, H. Liu, D. Li, and R. Yang, "Study on VRP in express distribution based on genetic algorithm," Logistics Technology, no. 3, pp. 101-105, 2013

[4] X. Hu, N. Yu, and Q. Ding, "Sequential decision methods for disruption management in distribution," Journal of Industrial Engineering and Engineering Management, vol. 25, no. 2, pp. 186-190, 2011.

[5] B. B. Li, and L. Wang, "A hybrid quantum-inspired genetic algorithm for multi-objective flow shop scheduling," IEEE Transactions on Systems, Man and Cybemetics, vol. 37, no. 3, pp. 576-591, 2007.

[6] J. Chen, "Study on routing optimization for physical distribution based on ant colony algorithm," Computer Simulation, vol. 22, no. 2, pp. 268-271, 2011.

[7] Z. Li, "Mixed ant colony algorithm solving the VRP problem," Journal of Wuhan University of Technology (Transportation Science \& Engineering), vol. 30, no. 2, pp. 306-309, 2006.

[8] F. Zhang, and S. Wang, "Study on data analysis oriented route programming algorithm in logistics system," Logistics Technology, no. 6, pp. 173-176, 2013

[9] L. Li, and W. Jiang, "Study on application of ant colony genetic optimization algorithm in choosing logistics distribution routes," Journal of Harbin University of Commerce (Natural Sciences Edition), vol. 25, no. 6, pp. 707-710, 2009.

[10] T. Wang, and Y. Wu, "Study on optimization of logistics distribution route based on chaotic PSO," Computer Engineering and Applications, vol. 47, no. 29, pp. 218-221, 2011.

Received: February 26, 2015

Revised: April 29, 2015

Accepted: May 20, 2015

(c) Xiao-jun and Bin; Licensee Bentham Open.

This is an open access article licensed under the terms of the Creative Commons Attribution Non-Commercial License (http://creativecommons.org/licenses/by-nc/3.0/) which permits unrestricted, non-commercial use, distribution and reproduction in any medium, provided the work is properly cited. 